Research Article

\title{
Theoretical Investigation of an Air-Slot Mode-Size Matcher between Dielectric and MDM Plasmonic Waveguides
}

\author{
Rami A. Wahsheh \\ Communications Engineering Department, Princess Sumaya University for Technology, Amman, Jordan \\ Correspondence should be addressed to Rami A. Wahsheh; r.wahsheh@psut.edu.jo
}

Received 27 October 2021; Accepted 3 December 2021; Published 14 December 2021

Academic Editor: Rujiang Li

Copyright (C) 2021 Rami A. Wahsheh. This is an open access article distributed under the Creative Commons Attribution License, which permits unrestricted use, distribution, and reproduction in any medium, provided the original work is properly cited.

Hybrid integration of dielectric and plasmonic waveguides is necessary to reduce the propagation losses due to the metallic interactions and support of nanofabrication of plasmonic devices that deal with large data transfer. In this paper, we propose a direct yet efficient, very short air-slot coupler (ASC) of a length of $36 \mathrm{~nm}$ to increase the coupling efficiency between a silicon waveguide and a silver-air-silver plasmonic waveguide. Our numerical simulation results show that having the ASC at the interface makes the fabrication process much easier and ensures that light couples from a dielectric waveguide into and out of a plasmonic waveguide. The proposed coupler works over a broad frequency range achieving a coupling efficiency of $86 \%$ from a dielectric waveguide into a metal-dielectric-metal (MDM) plasmonic waveguide and 68\% from a dielectric waveguide to an MDM plasmonic waveguide and back into another dielectric waveguide. In addition, we show that even if there are no high-precision fabrication techniques, light couples from a conventional dielectric waveguide (CDW) into an MDM plasmonic waveguide as long as there is an overlap between the CDW and ASC, which reduces the fabrication process tremendously. Our proposed coupler has an impact on the miniaturization of ultracompact nanoplasmonic devices.

\section{Introduction}

Efficient coupling of light into a metal-dielectric-metal $(\mathrm{MDM})$ waveguide from a conventional dielectric waveguide $(\mathrm{CDW})$ is the future of the on-chip applications of the plasmonic devices such as splitters $[1,2]$, Mach-Zehnder interferometers [3, 4], reflectors [5], wavelength demultiplexers [6], circulators [7], filters [8], and all-optical switching [9]. Other methods of controlling the flow of light in subwavelength regime involve using phase-change materials (PCMs) such as germanium-antimony-telluride $\left(\mathrm{Ge}_{2} \mathrm{Sb}_{2} \mathrm{Te}_{5}\right)$ that achieve fast switching speeds by controlling their index of refraction. In $[10,11]$, the authors experimentally demonstrated the integration of a PCM with hybrid metal-dielectric metasurfaces to control the amplitude, phase, and polarization of the incident optical wavefront. In our research paper, the low-loss CDWs are used to couple light into and out of the plasmonic waveguides that have large propagation losses due to their large metallic interactions. Several different structures have been proposed to achieve mode matching between a CDW and an MDM plasmonic waveguide [12-20]. These solutions are not practical to fabricate or too long for ultracompact integrated circuits. One solution in the literature that has attracted a lot of attention is proposed using a very compact air-gap coupler (AGC) at the interface between a MDM plasmonic waveguide and CDW [21]. The proposed AGC provided a large fabrication tolerance in addition to high transmission coupling efficiency (TCE) into the output dielectric waveguide. Another solution that attracted a lot of attention, because of its simple and compact design, is the air-slot coupler (ASC) that we proposed in [22]. The proposed ASC was fabricated between a $460 \mathrm{~nm}$-wide silicon waveguide and $80 \mathrm{~nm}$-wide gold-air-gold plasmonic waveguide. The TCE was about $40 \%$ when the length of the MDM waveguide was $500 \mathrm{~nm}$. The proposed ASC couples light from the CDW into the ASC waveguide before it is coupled into the MDM plasmonic waveguide. The air-slot waveguide that is located inside the CDW at the interface with the MDM plasmonic waveguide is the ASC. Having the ASC at the interface with the CDW ensures that light couples from the CDW into and out of the MDM plasmonic waveguide. In this paper, we 
show the design steps and numerical results of a very compact ASC which is smaller than that reported in our previous work in [22], has better TCE into the output CDW, and requires the use of less-precision fabrication techniques. Our proposed ASC is between a $300 \mathrm{~nm}$-wide silicon waveguide and a $40 \mathrm{~nm}$-wide silver-air-silver plasmonic waveguide. In addition, we show the sensitivity of our designs to different fabrication challenges.

The proposed ASCs were designed and analyzed using a two-dimensional (2D) finite-difference time-domain method. We used a uniform mesh size of $1 \mathrm{~nm}$ to accurately capture the changes of the field at the interface of the CDW, ASC, and MDM plasmonic waveguides. The dielectric material of the CDW is silicon, and that of the metal is silver. The TCE in our analysis is calculated by normalizing the output power measured at the output CDW with respect to the input power of the launched light, while the $\mathrm{CE}$ in our analysis is calculated by normalizing the power measured inside the MDM plasmonic waveguide with respect to the input power of the launched light. The propagation losses of the MDM plasmonic waveguides are included in our numerical results, while those of the CDWs are not included to show the effect of using the ASC on the CE.

The remainder of this paper is organized as follows: in Section 2, we explain the designs and results of the ASC. We also show how increasing the width of the CDW can increase the CE into the MDM plasmonic waveguide. In Section 3, we compare the spectrum results of the proposed ASCs. In Section 4, we show how our proposed designs increase the fabrication tolerance which is needed when aligning the dielectric waveguide to the plasmonic waveguide. Finally, in Section 5, we provide conclusions.

\section{Air-Slot Coupler Design, Analysis, and Fabrication}

Figure 1(a) shows the schematics of our proposed ASC \#1. The ASC is located inside the CDW at the interface between a $300 \mathrm{~nm}$-wide CDW and a $40 \mathrm{~nm}$-wide MDM plasmonic waveguide. The dependence of the $\mathrm{CE}$ on the length of the ASC, $L_{\mathrm{ASC}}$, is shown in Figure 1(b). As $L_{\mathrm{ASC}}$ increases, the $\mathrm{CE}$ oscillates and light continues to couple from the CDW into the MDM plasmonic waveguide. The CE increased from $68 \%$ to $80 \%$ as $L_{\mathrm{ASC}}$ increased from zero (i.e., butt coupling) to $36 \mathrm{~nm}$, respectively. As shown in Figure 1(b), almost zero back reflection resulted when $L_{\mathrm{ASC}}=36 \mathrm{~nm}$ which means that the ASC reduced the mode-size mismatch between the size of the mode in the CDW and that in the slot waveguide (i.e., the ASC and the MDM plasmonic waveguide). As long as there is an overlap between the slot waveguide and the CDW, light continues to couple into the MDM plasmonic waveguide. This reduces the fabrication complexity especially when using the focused ion beam (FIB) to define both ASC and MDM plasmonic waveguide.

Moreover, we found that the CE of ASC \# 1 can be further improved by increasing the width of the CDW, $W_{\mathrm{Si}}$, over a length of $L_{\mathrm{Si}}$ before it is connected to the MDM plasmonic waveguide (the new design is called ASC \#2; see Figure 1(c)). As shown in Figure 1(d), the CE of ASC \#2 increased from $80 \%$ to about $86 \%$ when $W_{\mathrm{Si}}$ increased from $300 \mathrm{~nm}$ to $360 \mathrm{~nm}$ over a length of $L_{\mathrm{Si}}=360 \mathrm{~nm}$. Even though the back reflection of ASC \#2 increased slightly to $0.025 \%$ compared to $0 \%$ in ASC \#1, expanding the width of the CDW acted as a fine-tuning mode size to match the mode size inside the $\mathrm{CDW}$ to that in the slot waveguide.

To study the TCE from a $300 \mathrm{~nm}$-wide CDW into a $40 \mathrm{~nm}$-wide MDM plasmonic waveguide and back into a $300 \mathrm{~nm}$-wide CDW, the MDM plasmonic waveguide with the ASC is embedded between two CDWs. ASC \#1 is connected back to back with another ASC \#1 (the new design is called ASC \#3; see Figure 2(a)), and ASC \#2 is connected back to back with another ASC \#2 (the new design is called ASC \#4; see Figure 2(b)). In both designs, ASCs \#3 and \#4, the ASC of a length of $36 \mathrm{~nm}$ is placed at each end of the MDM plasmonic waveguide. In order to find the optimum design dimensions, we compared the dependence of the TCE of ASC \#3 with that of ASC \#4 as a function of the length of the MDM waveguide, $L_{\mathrm{MDM}}$; see Figure $2(\mathrm{c})$. As shown in Figure 2(c), the oscillations in the measured TCE occurred due to the slot waveguide behaving as a Fabry-Perot (FP) cavity-like structure. As $L_{\mathrm{MDM}}$ increases, the TCE decreases due to the metallic propagation losses in the MDM plasmonic waveguide. Less oscillations with higher TCE are achieved by using ASC \#4. Both designs, ASCs \#3 and \#4, achieved higher TCE than that reported in [22]. The field distributions of the coupled light at $1550 \mathrm{~nm}$ for ASCs \#3 and \#4 are shown in Figures 2(d) and 2(e) when $L_{\mathrm{MDM}}=740 \mathrm{~nm}$.

ASC \#4 could be fabricated on a $250 \mathrm{~nm}$ silicon-oninsulator wafer. First, the alignment marks are defined, and then the dielectric waveguides are placed at specific locations from the alignment marks, followed by etching the silicon layer at the areas where the silver layer is defined. Then, a platinum layer is deposited on top of the silver layer before using the FIB to define the air-slot waveguides.

\section{Spectrum Analysis}

The spectrum of the proposed ASCs \#3 and \#4 is shown in Figure 3. The wavelength varied from $400 \mathrm{~nm}$ to $2400 \mathrm{~nm}$ in steps of $25 \mathrm{~nm}$. At each step, the TCE into the output CDW was measured. The length of the MDM plasmonic waveguide, $L_{\mathrm{MDM}}$, was chosen as $740 \mathrm{~nm}$ which represents the second peak in Figure 2(c). The spectrum of the butt coupling with and without applying the $W_{\mathrm{Si}}$ width expansion (i.e., $L_{\mathrm{ASC}}=0 \mathrm{~nm}$ ) was also added to Figure 3 to show that using the ASC increases the TCE around the communication wavelength of $1550 \mathrm{~nm}$ for the two proposed couplers. In comparing the spectrum of ASCs \#3 and \#4, we found that tapering the CDW not only resulted in a higher TCE into the output CDW but also shifted the spectrum response by about $100 \mathrm{~nm}$ to the right.

Our proposed ASC \#4 with the $W_{\mathrm{Si}}$ width expansion can be used in different sensing applications to show that the refractive index of the slot waveguide, $n_{\text {Slot }}$ (i.e., the dielectric in the MDM plasmonic waveguide in addition to that in the 


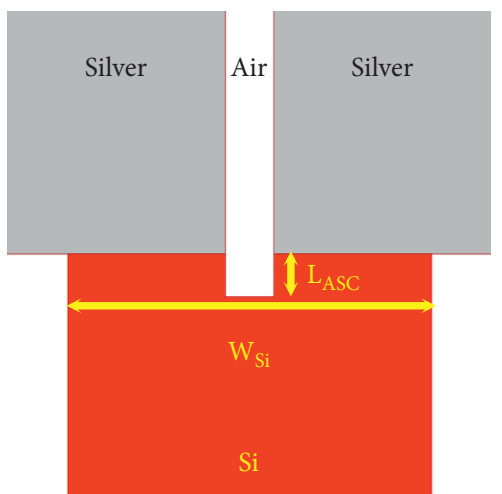

(a)

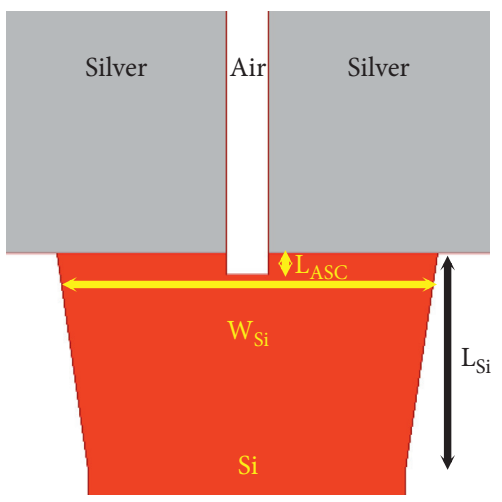

(c)

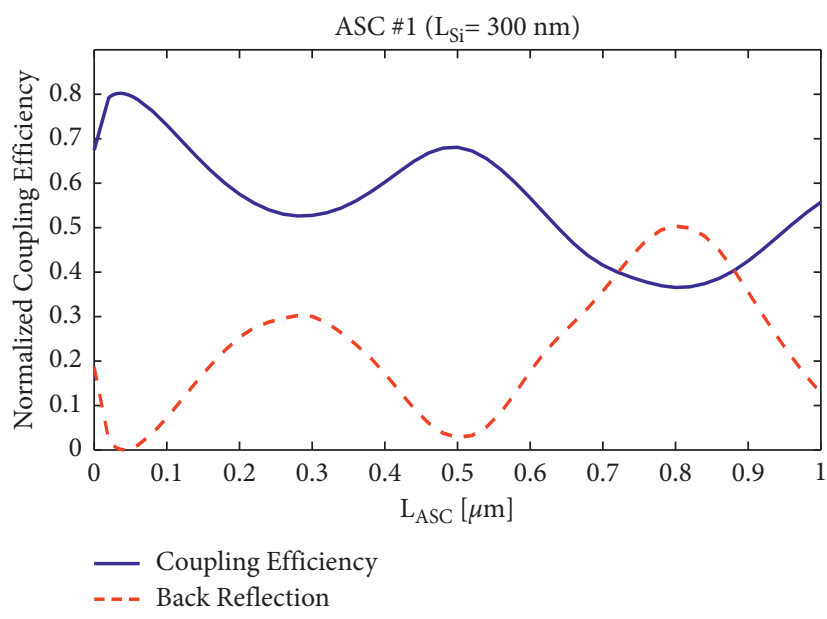

(b)

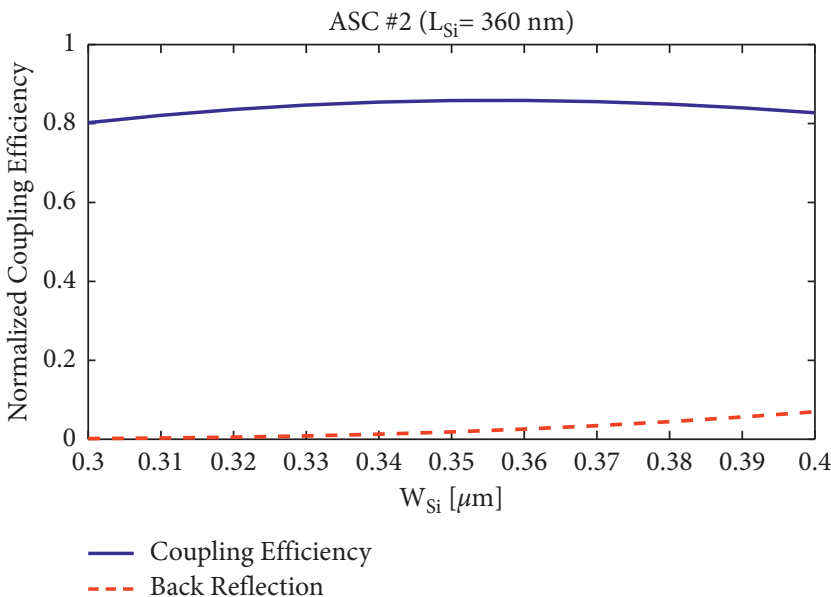

(d)

FIgURE 1: The schematics of the proposed ASCs \#1 and \#2 that show how light couples into the MDM plasmonic waveguide: (a) ASC \#1 without the dielectric width expansion, (b) coupling efficiency as a function of the length of the ASC, $L_{\mathrm{ASC}}$, for ASC \#1, (c) ASC \#2 with the dielectric width expansion, and (d) coupling efficiency as a function of the width of the CDW, $W_{\mathrm{Si}}$, for ASC \#2.

ASC) was changed from 1 (air) to 3.5 (silicon) in steps of 0.5 while keeping $L_{\mathrm{MDM}}$ at $740 \mathrm{~nm}$ (as shown in Figure 4). As $n_{\text {Slot }}$ increases, the TCE decreases, and the shape of the spectrum changes as a result of the poor coupling from the $\mathrm{CDW}$ into the slot waveguide in addition to the oscillations in the slot waveguide that behaved like an FP cavity-like structure. The TCE value is zero from about $950 \mathrm{~nm}$ to about $1150 \mathrm{~nm}$ for all types of $n_{\text {Slot }}$.

\section{Analysis of the Sensitivity of the Design to Different Fabrication Challenges}

We investigated the effect of changing various parameters of the design on the spectrum response of our proposed ASC \#4. The investigated parameters are shown in Figure 5(a): the length of the air-slot coupler inside the silicon waveguide, $L_{\mathrm{ASC}}$; the length of the MDM plasmonic waveguide, $L_{\mathrm{MDM}}$; the width of the silicon waveguide, $W_{\mathrm{Si}}$; the width of the slot waveguide, $W_{\text {Slot }}$ and the misalignment between the position of the MDM plasmonic waveguide with respect to the center of the silicon waveguide, $S$. We changed one parameter at a time and studied its effect on the spectrum response. The used optimum values for $L_{\mathrm{ASC}}, L_{\mathrm{MDM}}, W_{\mathrm{Si}}, W_{\text {Slot }}$, and $S$ were $36 \mathrm{~nm}, 740 \mathrm{~nm}, 360 \mathrm{~nm}, 40 \mathrm{~nm}$, and $0 \mathrm{~nm}$, respectively. We found that changing $L_{\mathrm{ASC}}$ from $0 \mathrm{~nm}$ (i.e., butt coupling) to $400 \mathrm{~nm}$ resulted in a reduction in the width of the spectrum response mainly due to the FP cavity-like structure; see Figure 5(b). The highest TCE with the largest spectrum width occurred when $L_{\mathrm{ASC}}=36 \mathrm{~nm}$. Three lengths of $L_{\mathrm{MDM}}$ were investigated, $220 \mathrm{~nm}, 560 \mathrm{~nm}$, and $740 \mathrm{~nm}$, which represent the first peak, the first valley, and the second peak in Figure 2(a), respectively. We found that almost no change occurred in the spectrum shape when $L_{\mathrm{MDM}}$ is changed except for the reduction in the TCE due to the metallic interactions; see Figure 5(c). We also found that the cutoff wavelength can be controlled by changing $W_{\mathrm{Si}}$ (see Figure $5(\mathrm{~d})$ ) and $W_{\text {Slot }}$ (see Figure 5(e)). Changing $W_{\mathrm{Si}}$ from $360 \mathrm{~nm}$ to $440 \mathrm{~nm}$ resulted in shifting the cutoff wavelength from about $1100 \mathrm{~nm}$ to $1300 \mathrm{~nm}$, respectively, whereas changing $W_{\text {Slot }}$ from $40 \mathrm{~nm}$ to $120 \mathrm{~nm}$ resulted in shifting the cutoff wavelength from 


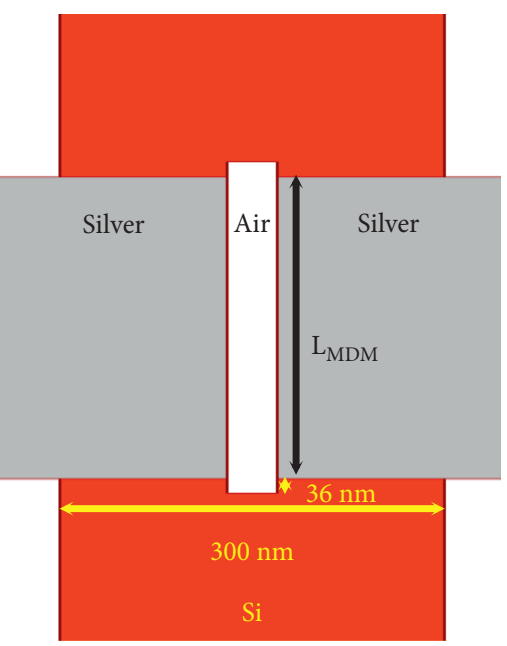

(a)

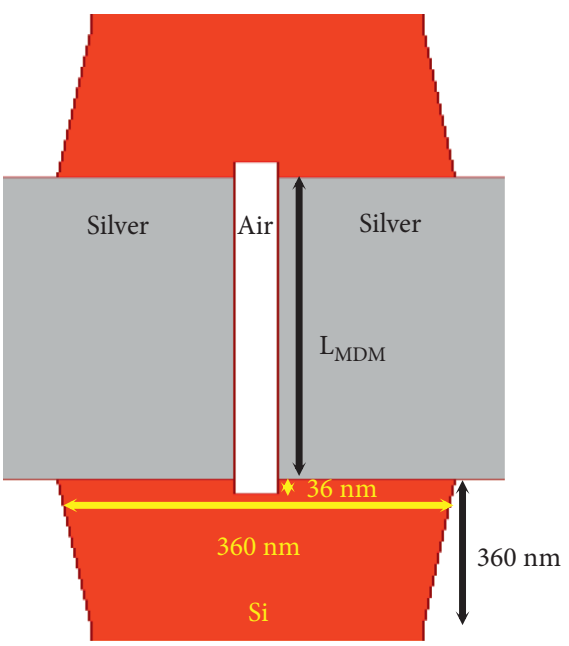

(b)

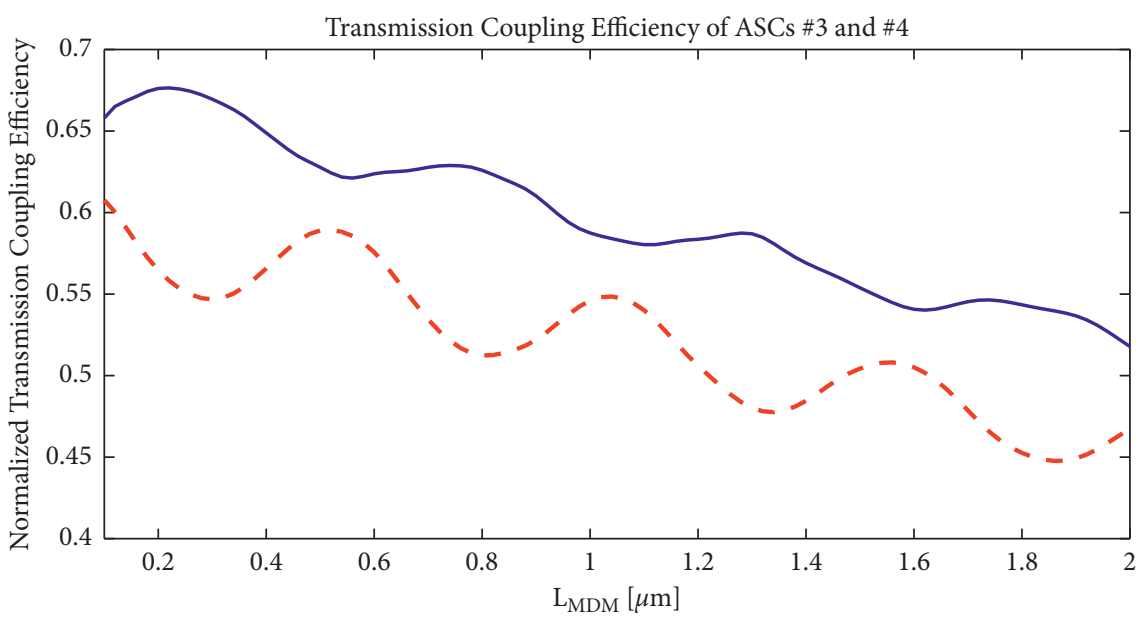

ASC \#4

- - - ASC \#3

(c)

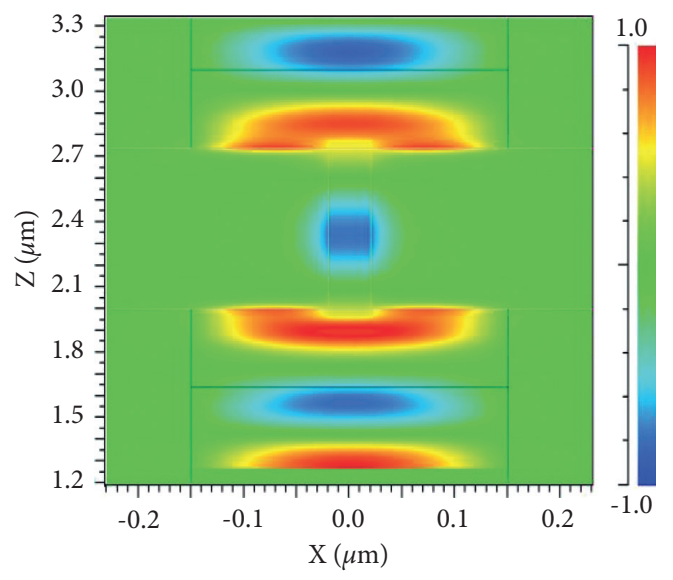

(d)

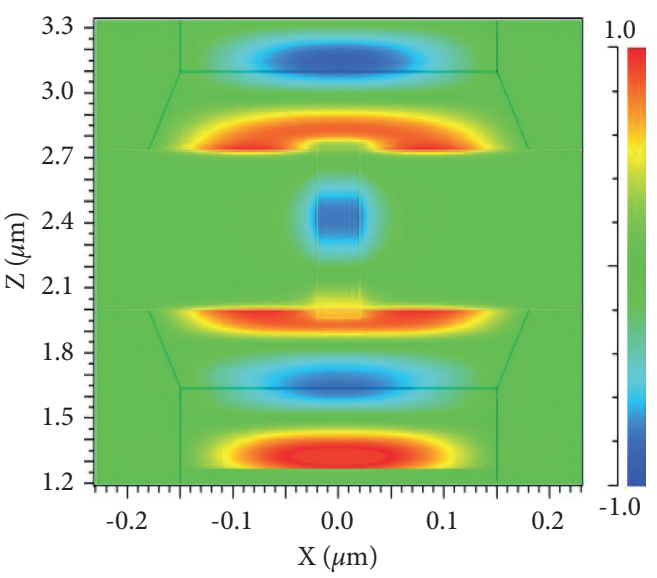

(e)

FIgURE 2: The schematics of the proposed ASCs \#3 and \#4 that show how light couples into and out of the MDM plasmonic waveguide: (a) ASC \#3 without the dielectric width expansion that resulted from connecting two \#1 ASCs back to back, (b) ASC \#4 with the dielectric width expansion that resulted from connecting two \#2 ASCs back to back, (c) transmission coupling efficiency as a function of the length of the MDM plasmonic waveguide, $L_{\mathrm{MDM}}$, for both ASCs \#3 and \#4, and (d, e) field distributions of the coupled light at $1550 \mathrm{~nm}$ for ASCs \#3 and \#4 when $L_{\mathrm{MDM}}=740 \mathrm{~nm}$. 


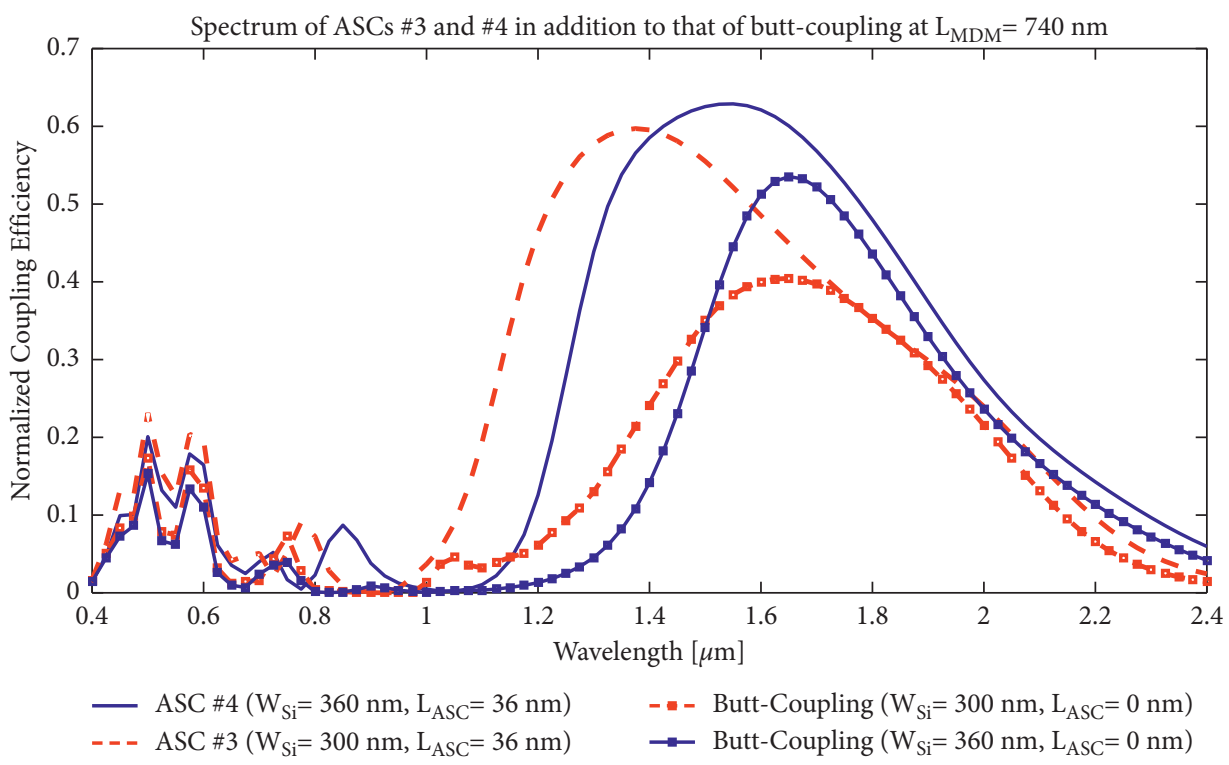

FIGURE 3: The spectrum of the proposed ASCs \#3 and \#4 in addition to that of butt coupling with and without the $W_{\mathrm{Si}}$ width expansion.

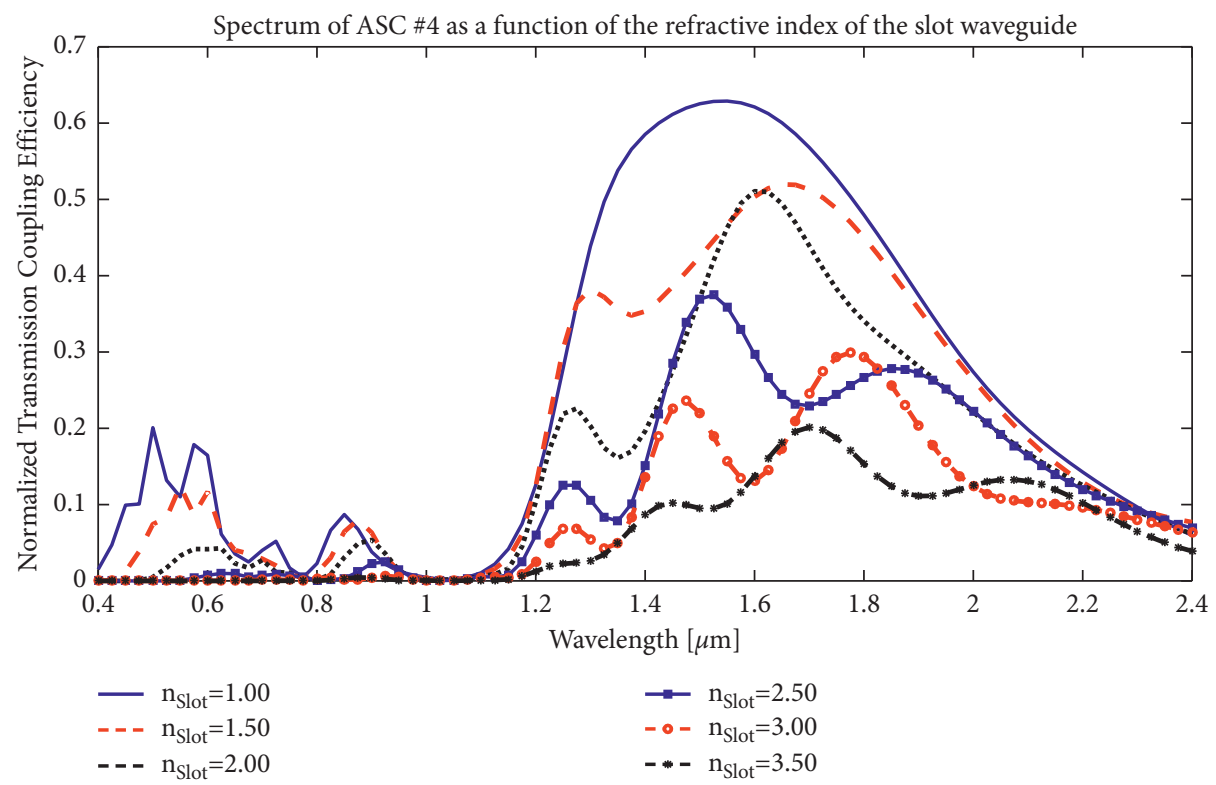

FIGURE 4: The spectrum of ASC \#4 for different refractive indices of the slot waveguide, $n_{\text {Slot }}$.

about $1100 \mathrm{~nm}$ to $1000 \mathrm{~nm}$, respectively. Finally, we found that changing $S$ from $0 \mathrm{~nm}$ to $80 \mathrm{~nm}$ resulted in a reduction in the width of the spectrum response in addition to a reduction in the TCE due to the reduction in the overlapped area between the mode supported by the slot waveguide and that supported by the silicon waveguide; see Figure 5(f). This analysis shows the impact of different key design parameters on the spectrum response of the device which indicates that using the ASC reduces the need for high-precision fabrication process tremendously. 

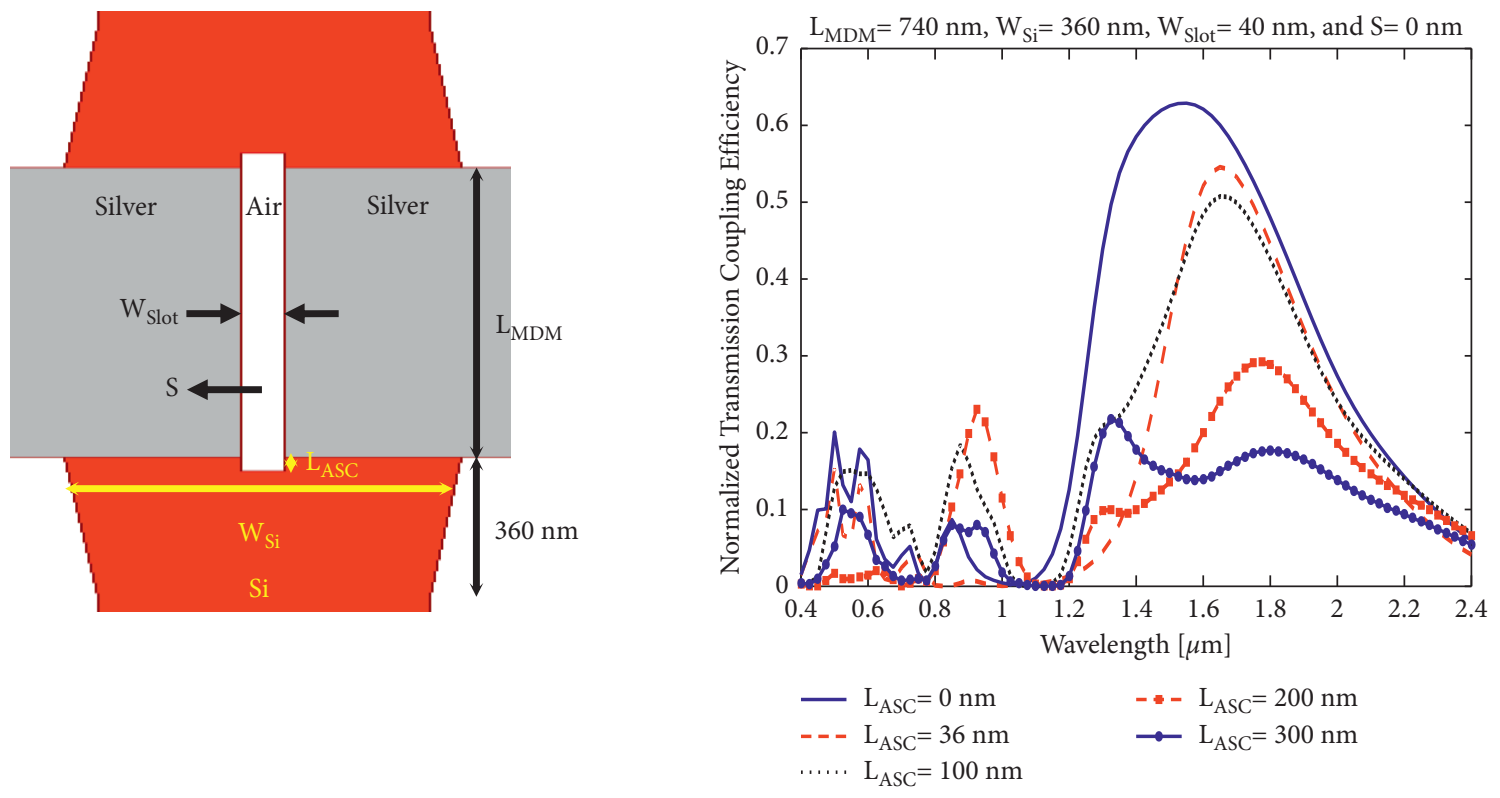

(a)

(b)
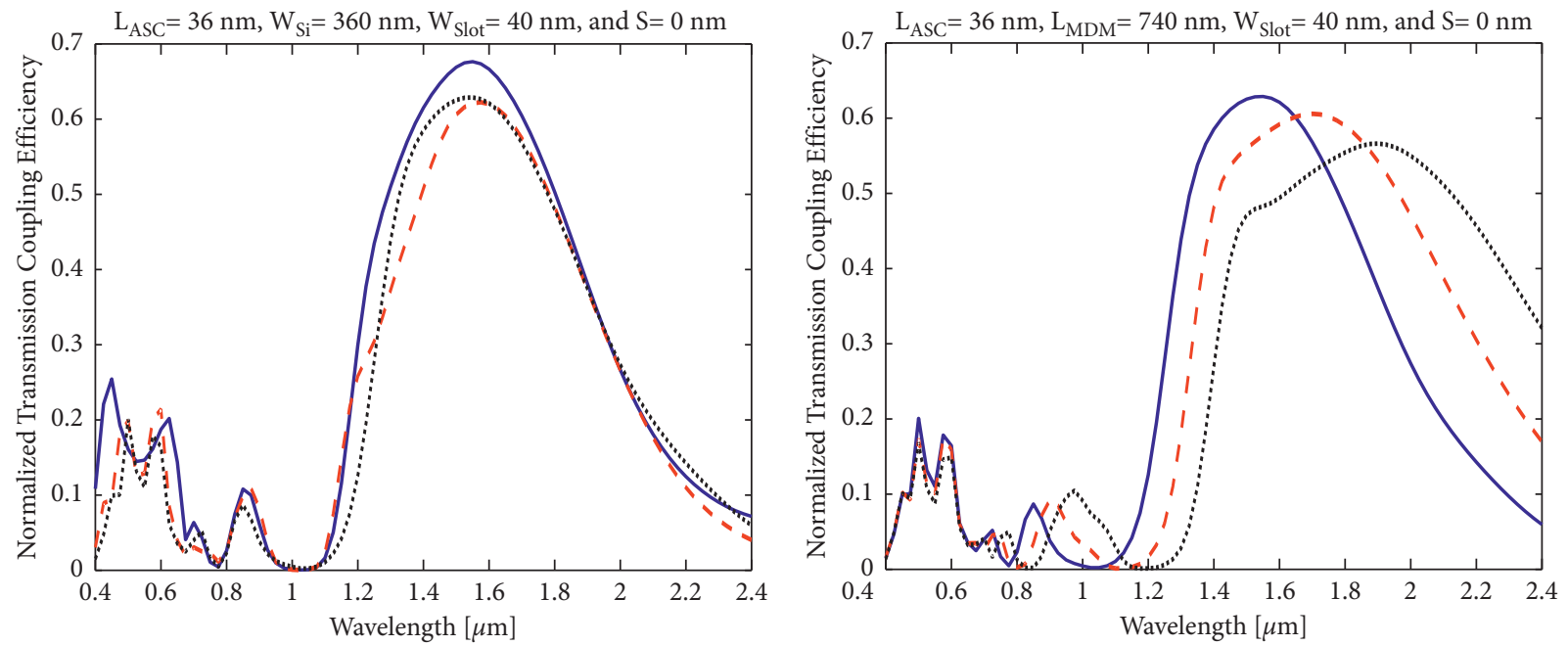

- $\mathrm{L}_{\mathrm{MDM}}=220 \mathrm{~nm}$
- - $\mathrm{L}_{\mathrm{MDM}}=560 \mathrm{~nm}$
$\ldots \ldots \mathrm{L}_{\mathrm{MDM}}=740 \mathrm{~nm}$

- $\mathrm{W}_{\mathrm{Si}}=360 \mathrm{~nm}$

- - - $\mathrm{W}_{\mathrm{Si}}=400 \mathrm{~nm}$

...... $\mathrm{W}_{\mathrm{Si}}=440 \mathrm{~nm}$

(c)

(d)

Figure 5: Continued. 


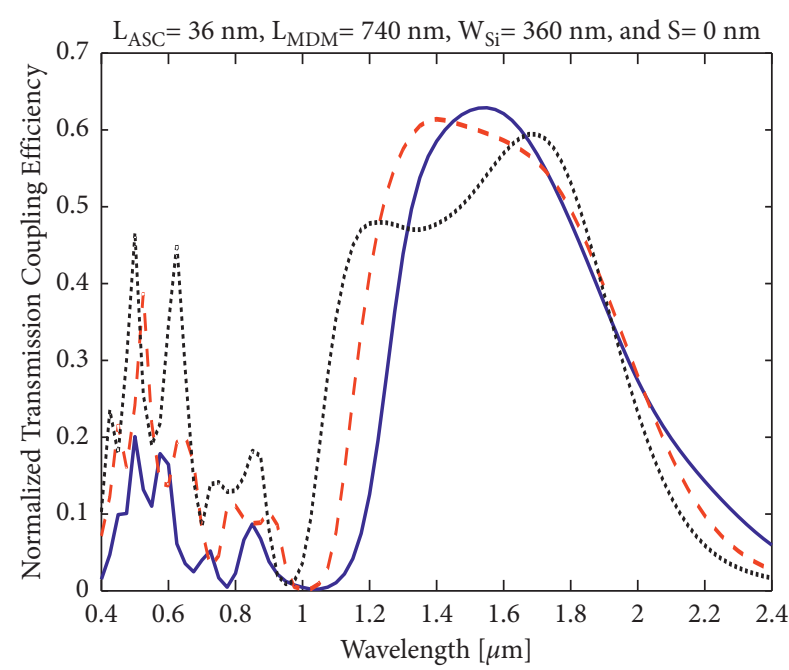

$-\mathrm{W}_{\text {Slot }}=40 \mathrm{~nm}$
$---\mathrm{W}_{\text {Slot }}=80 \mathrm{~nm}$
$\ldots \ldots \mathrm{W}_{\text {Slot }}=120 \mathrm{~nm}$

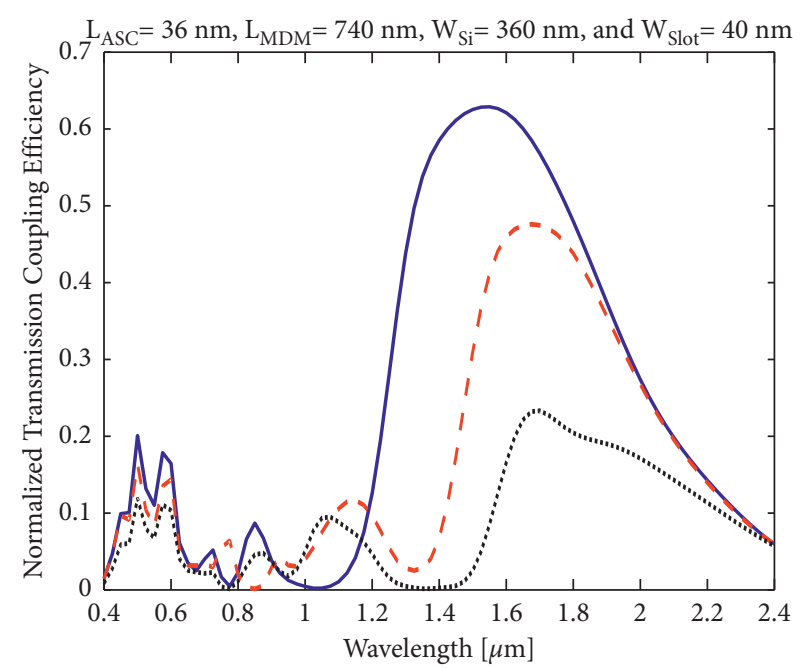

$\ldots \mathrm{S}=0 \mathrm{~nm}$
$-\ldots \mathrm{S}=40 \mathrm{~nm}$
$\ldots . \mathrm{S}=80 \mathrm{~nm}$

(e)

(f)

Figure 5: (a) A schematic of our proposed ASC \#4 that shows the design parameters and (b-f) dependence of the spectrum response of ASC $\# 4$ on the length of the air-slot coupler $L_{\mathrm{ASC}}$, the length of the MDM plasmonic waveguide $L_{\mathrm{MDM}}$, the width of the silicon waveguide $W_{\mathrm{Si}}$, the width of the slot waveguide $W_{\text {Slot }}$, and the misalignment between the position of the air-slot waveguide with respect to the center of the silicon waveguide $S$, respectively.

\section{Conclusions}

We numerically showed that a very short ASC of a length of $36 \mathrm{~nm}$ and a width of $40 \mathrm{~nm}$ can be used to couple light from a $40 \mathrm{~nm}$-wide silicon waveguide into a $40 \mathrm{~nm}$-wide MDM plasmonic waveguide. When changing the key design parameters (i.e., the width of the silicon waveguide; the length of the MDM plasmonic waveguide; and the length, width, and position of the ASC), our results indicated that using our proposed ASC reduced the need for high-precision fabrication process tremendously. Moreover, the spectrum of the proposed ASC operated at a broad frequency range around the communication wavelength of $1550 \mathrm{~nm}$.

\section{Data Availability}

I did many simulations using the FDTD method to get all the results that I showed in the submitted research work. I described all the required information that one needs to redo the same design so that he/she gets the same results.

\section{Conflicts of Interest}

The author declares that there are no conflicts of interest.

\section{References}

[1] R. A. Wahsheh, "Ultra-compact broadband 3-dB metal-dielectric-metal plasmonic power splitter," Journal of Modern Optics, vol. 68, no. 3, pp. 153-160, 2021.

[2] X. Shi, W. Yang, H. Xing, and X. Chen, "Design of power splitters based on hybrid plasmonic waveguides," Applied Sciences, vol. 11, no. 18, Article ID 8644, 2021.
[3] R. A. Wahsheh, Z. Lu, and M. A. G. Abushagur, "Nanoplasmonic directional couplers and Mach-Zehnder interferometers," Optics Communications, vol. 282, no. 23, pp. 4622-4626, 2009.

[4] S. A. Maragheh, B. Olyaeefar, and R. Kheradmand, "Ultrafast nano-scale optical switching in a plasmonic interferometer with enhanced tunability," Plasmonics, vol. 15, no. 2, pp. 435-439, 2020.

[5] B. Wang and G. P. Wang, "Plasmon Bragg reflectors and nanocavities on flat metallic surfaces," Applied Physics Letters, vol. 87, no. 1, Article ID 013107, 2005.

[6] H. Lu, X. Liu, Y. Gong, D. Mao, and L. Wang, "Enhancement of transmission efficiency of nanoplasmonic wavelength demultiplexer based on channel drop filters and reflection nanocavities," Optics Express, vol. 19, no. 14, pp. 12885-12890, 2011.

[7] K. Wen, L. Yan, W. Pan, B. Luo, Z. Guo, and Y. Guo, “A fourport plasmonic quasi-circulator based on metal-insulatormetal waveguides," Optics Express, vol. 20, no. 27, pp. 28025-28032, 2012.

[8] Y. Gong, X. Liu, and L. Wang, "High-channel-count plasmonic filter with the metal-insulator-metal Fibonacci-sequence gratings," Optics Letters, vol. 35, no. 3, pp. 285-287, 2010.

[9] G. Wang, H. Lu, X. Liu, and Y. Gong, "Numerical investigation of an all-optical switch in a graded nonlinear plasmonic grating," Nanotechnology, vol. 23, no. 44, Article ID 444009, 2012.

[10] S. Abdollahramezani, O. Hemmatyar, M. Taghinejad et al., "Dynamic hybrid metasurfaces," Nano Letters, vol. 21, no. 3, pp. 1238-1245, 2021.

[11] S. Abdollahramezani, O. Hemmatyar, H. Taghinejad et al., "Tunable nanophotonics enabled by chalcogenide phasechange materials," Nanophotonics, vol. 9, no. 5, pp. 1189-1241, 2020. 
[12] P. Ginzburg, D. Arbel, and M. Orenstein, "Gap plasmon polariton structure for very efficient microscale-to-nanoscale interfacing," Optics Letters, vol. 31, no. 22, pp. 3288-3290, 2006.

[13] S. H. Badri and M. M. Gilarlue, "Coupling between silicon waveguide and metal-dielectric-metal plasmonic waveguide with lens-funnel structure," Plasmonics, vol. 15, no. 3, pp. 821-827, 2020.

[14] G. Veronis and S. Fan, "Theoretical investigation of compact couplers between dielectric slab waveguides and two-dimensional metal-dielectric-metal plasmonic waveguides," Optics Express, vol. 15, no. 3, pp. 1211-1221, 2007.

[15] C.-T. Chen, X. Xu, A. Hosseini, Z. Pan, and R. T. Chen, "High efficiency silicon strip waveguide to plasmonic slot waveguide mode converter," Optical Interconnects XV, vol. 9368, Article ID 936809, 2015.

[16] D. Kong and M. Tsubokawa, "Evaluation of slot-to-slot coupling between dielectric slot waveguides and metal-insulator-metal slot waveguides," Optics Express, vol. 23, no. 15, pp. 19082-19091, 2015.

[17] V. Patel, P. Sharma, and V. D. Kumar, "Efficient coupling from dielectric to hybrid plasmonic waveguide using curved taper," IEEE Photonics Technology Letters, vol. 31, no. 4, pp. 323-326, 2019.

[18] Z. Han, A. Y. Elezzabi, and V. Van, "Experimental realization of subwavelength plasmonic slot waveguides on a silicon platform," Optics Letters, vol. 35, no. 4, pp. 502-504, 2010.

[19] L. Chen, J. Shakya, and M. Lipson, "Subwavelength confinement in an integrated metal slot waveguide on silicon," Optics Letters, vol. 31, no. 14, pp. 2133-2135, 2006.

[20] P. Sun, P. Xu, K. Zhu, and Z. Zhou, "Silicon-Based optoelectronics enhanced by hybrid plasmon polaritons: bridging dielectric photonics and nanoplasmonics," Photonics, vol. 8, no. 11, p. 482, 2021.

[21] R. A. Wahsheh, "Mode coupling enhancement from dielectric to plasmonic waveguides," Optical Engineering, vol. 59, no. 10, Article ID 107101, 2020.

[22] R. A. Wahsheh and M. A. G. Abushagur, "Experimental and theoretical investigations of an air-slot coupler between dielectric and plasmonic waveguides," Optics Express, vol. 24, no. 8, pp. 8237-8242, 2016. 